\title{
Tetramethylpyrazine improved the survival of multiterritory perforator flaps by inducing angiogenesis and suppressing apoptosis via the Akt/Nrf2 pathway
}

This article was published in the following Dove Press journal:

Drug Design, Development and Therapy

\author{
LiMing Qing ${ }^{1,2, *}$ \\ PanFeng $\mathrm{Wu}^{\mathrm{l}, *}$ \\ ZhengBing Zhou' \\ Fang $\mathrm{Yu}^{\prime}$ \\ JuYu Tang'
}

'Department of Orthopedics, Xiangya Hospital, Central South University, Changsha, Hunan, People's Republic of China; ${ }^{2}$ Department of Neurosurgery, Maryland University of Medicine SchoolBaltimore, Baltimore, MD, USA

*These authors contributed equally to this work
Correspondence: JuYu Tang

Department of Orthopaedics, Xiangya Hospital, Central South University, 87

Xiangya Road, Changsha, Hunan 410008,

People's Republic of China

Email tangjuyu7749@csu.edu.cn
Background: Multiterritory perforator flaps were commonly designed to cover the large soft-tissue defects in reconstructive surgery. But the high risk of partial necrosis in the distal portion of the flaps hindered their clinical application. The purpose of this study was to evaluate the effects of tetramethylpyrazine (TMP) on the survival of the multiterritory perforator flaps and to explore the underlying mechanism.

Materials and methods: Seventy-two Sprague-Dawley rats underwent multiterritory perforator flap procedure and were divided into three groups with 24 each. Flap survival and water content were measured, and the area of angiogenesis and apoptosis in the ischemia skin flaps were assessed on the postoperative day 7. The expressions of angiogenesis-related protein VEGF and apoptosis-related protein Bax, Bcl-2 in each group were detected by Western blotting, which also had been used to assess the expressions levels of Akt, p-Akt, and Nrf2.

Results: Following TMP treatment, the survival area and number of microvessels presented in the skin flaps increased and tissue edema reduced on postoperative day 7 . The expressions of angiogenesis-related protein VEGF increased in the TMP treatment group than in the control group. In addition, compared with the control group, TMP inhibited apoptosis, and increased the expression levels of p-Akt, Nrf2 in the areas of ischemia. These effects were reversed by an Akt protein inhibitor LY294002. Similarly, treatment with LY294002 inhibited TMP induced by interfering the Akt/Nrf2 signaling pathway.

Conclusion: These results illustrated that TMP could promote the survival of multiterritory perforator flaps by enhancing angiogenesis and attenuating apoptosis. These were involved in Akt/Nrf2 signaling pathway.

Keywords: perforator flaps, tetramethylpyrazine, apoptosis, angiogenesis, Akt/Nrf2 signaling pathway

\section{Introduction}

Multiterritory perforator flaps are one of the most popular procedure for reconstructive surgeons to repair soft-tissue defects caused by trauma or tumor ablation. ${ }^{1-5}$ However, practice clinical application often encounters dilemma because of complication on ischemic partial necrosis of the distal parts of the flaps. ${ }^{6}$ Our previous experience showed that skin flap necrosis may necessitate reoperation, prolong the length of hospital stay, and increase the treatment cost. ${ }^{4,5}$ Hence, improving the 
survival area of the flap has a vital clinical significance. Previous studies demonstrated that the survival of perforator flaps depends on the reliable blood supply and the timely and orderly angiogenesis. ${ }^{8-10}$ Recently, some studies also demonstrated that ischemia, and even necrosis, was associated with the oxidative stress. ${ }^{11,12}$ These detrimental events will further result in cellular apoptosis which causes persistent damage in the ischemia area of skin flaps. Therefore, inhibition of cellular apoptosis and acceleration of angiogenesis were the main strategies for promoting the survival area of multiterritory perforator flaps. ${ }^{7,13,14}$

Tetramethylpyrazine (TMP) is an important active ingredient of traditional Chinese medicines, and it is identified with several molecular targeting properties. ${ }^{15,16}$ In China, it is widely used to treat neurovascular disorders, 17 ischemic stroke, 18 myocardial infarction and spine cord injure $^{16}$ due to its effectiveness and low toxicity. Chen et $\mathrm{al}^{17}$ had shown that TMP exhibited a neuroprotective effect in vitro and in vivo through antioxidative stress properties and suppressing cell apoptosis. Some researchers had also described that TMP had a protective effect in vascular growth through unregulated VEGF signaling pathway. ${ }^{19}$ Emerging researches showed that the distal parts of multiterritory perforator flaps are prone to ischemia and necrosis; $;^{3,20}$ therefore, we hypothesized that TMP might improve flap survival, for the antioxidative stress properties of TMP and together with its ability to promote neovascularization and attenuate cell apoptosis.

On the basis of this background, we hypothesized that TMP treatment could prevent necrosis, increase angiogenesis, and attenuate cell apoptosis in the multiterritory perforator flaps. In the present study, we investigated whether TMP treatment could increase the survival of multiterritory perforator flaps in rat models and explored its relative molecular mechanism.

\section{Materials and methods}

\section{Ethics statement}

All manipulations and surgical procedures were performed in accordance with the guidelines of the China Council of Animal Care and with approval of the Central South University Committee on Laboratory Animals.

\section{Animals and groups}

Sprague-Dawley rats weighing 250-300 g were used. The rats were housed in the Animal Care Center of Central South of University (Changsha, Hunan, China) and provided with free access to food and water. Seventy-two rats were divided randomly into three groups: a saline group ( $\mathrm{n}=24$, control group), a TMP treatment group ( $\mathrm{n}$ =24), and a TMP + LY294002 treatment group ( $\mathrm{n}=24)$.

\section{Flap animal model}

In this study, the multiterritory perforator flap model was performed according to our previous study. ${ }^{21}$ Briefly, the rats were anesthetized with pentobarbital sodium anesthesia $(30 \mathrm{mg} / \mathrm{kg}$, intraperitoneal). The flap was raised by sharp dissection in the plane between the panniculus carnosus and the deep fascia. The flap was then sutured with 4-0 monofilament suture in the original position. The flap area contained three even vascular territories: proximal (iliolumbar vessel territory, Area I), intermediate (posterior interior intercostal vessel territory, Area II), and distal (thoracodorsal vessel territory, Area III). The size of the flap was approximately $4 \mathrm{~cm} \times 10 \mathrm{~cm}$ (Figure S1).

\section{Drug administration}

The TMP group was treated with $10 \mathrm{mg} / \mathrm{kg}$ TMP by daily intraperitoneal injections for 7 days after the operation. The control group received equal volumes of saline. In the TMP + LY294002 group, an intraperitoneal injection was performed with an Akt inhibitor LY294002 at a dosage of $10 \mathrm{mg} / \mathrm{kg} 30 \mathrm{mins}$ before TMP administration (dose) every time.

\section{Assessment of flap survival area}

The skin flap survival area was evaluated by digital image analysis. On postoperative day 7, the necrotic area (defined by dark color and scar formation) and total flap area were delineated. Surface areas were calculated using Image-Pro Plus Software (version 6.0, Media Cybernetics Inc., Bethesda, MD, USA) by an investigator who was blinded to the experimental groups. The skin flap survival results were expressed as a percentage of the surviving area relative to the total surface area of the flap.

\section{Tissue edema assessment}

The tissue edema was measured as it was described in the previous study. ${ }^{7,11}$ On postoperative day 7, the flap tissue specimens were weighed and then dehydrated in an autoclave at $50^{\circ} \mathrm{C}$. We weighed all specimens every day until the weight was stabilized for 2 days. The percentage of water content was calculated as follows: 
Tissue water content $=\frac{\text { Wet weight }- \text { Dry weight }}{\text { Wet weight }} \times 100 \%$

\section{Angiography}

On postoperative day 7 , six rats underwent whole-body angiography in each group according to the method described by our previous study. ${ }^{21}$ Briefly, $5 \mathrm{~g}$ of gelatin was diluted in $100 \mathrm{~mL}$ of tap water heated to $40^{\circ} \mathrm{C}$, and 80 $\mathrm{mg}$ of water-soluble red lead oxide was added. This mixture was injected into the rat's carotid artery until the rat's limbs turned red. After injection, the flaps were harvested and then fixed for $24 \mathrm{hrs}$ at $4^{\circ} \mathrm{C}$. The flaps were radiographed ( $55 \mathrm{kVp}, 25 \mathrm{~mA}, 20$-s exposure) with a soft X-ray machine (Fuji Computerized Radiography XG-1; Fujifilm, Tokyo, Japan).

\section{H\&E staining}

The tissue samples were excised from the middle part of Area II in each group and stored in $4 \%$ buffered formaldehyde solution. Formaldehyde-fixed samples were processed in paraffin and stained with H\&E using standard histology protocols. We calculated the number of microvessels per unit area under a light microscope $(\times 100 \mathrm{mag}$ nification), which indicated the microvascular density.

\section{Immunohistochemistry (IHC)}

Tissue sections stained with CD31 were used to evaluate microvessel density (MVD). Six sections in each group were deparaffinized in xylene and rehydrated through a graded set of ethanol baths. After washing, sections were blocked with $3 \%(\mathrm{v} / \mathrm{v}) \mathrm{H}_{2} \mathrm{O}_{2}$ and treated with $10.2 \mathrm{mM}$ sodium citrate buffer for 20 mins at $95^{\circ} \mathrm{C}$. After blocking with $10 \%(\mathrm{w} / \mathrm{v})$ bovine serum albumin PBS for $10 \mathrm{mins}$, the sections were incubated with antibody against CD31 $(1: 100)$ overnight at $4^{\circ} \mathrm{C}$. Slides were then rinsed in PBS and were incubated with horseradish peroxidase (HRP)conjugated secondary antibody and counterstained with hematoxylin. Flap tissues were imaged at $\times 20$ magnifications. Images were calculated with Image-Pro Plus software (Media Cybernetics, Rockville, MD, USA) for integral absorbance quantitation of CD31-positive blood vessels counting.

\section{Immunofluorescence staining}

The tissue specimens in each group were fixed $4 \%$ buffered formaldehyde solution. The slides were incubated with a primary antibody against TUNELS $(1: 100)$ at $4{ }^{\circ} \mathrm{C}$ overnight. Then, the slides were washed three times for 10 mins at room temperature and incubated with second antibody for $1 \mathrm{hr}$ at room temperature. The nucleus was stained with DAPI (Invitrogen, Carlsbad, CA, USA) for 15 mins. The staining slides were observed by fluorescent Nikon Ti-E inverted microscope (Nikon, Kobe, Japan). For quantification, five different fields from each sample preparation were randomly selected, and then the numbers of TUNELS-positive cell areas were analyzed using Image J software (Media Cybernetics).

\section{Western blotting analysis}

Tissue samples in each group were harvested from the middle of Area II and stored $-80^{\circ} \mathrm{C}$ before Western blot analysis. These samples were homogenized in lysis buffer. The membranes were blocked in PBS with $0.1 \%$ Tween20 (PBS-T) containing 5\% milk powder for 30 mins at room temperature and then incubated overnight at $4{ }^{\circ} \mathrm{C}$ with one of the following primary antibodies: VEGF (1:1,000), Bcl-2 (1:1,000), Bax (1:1,000), Nrf2 (1:1,000), $\operatorname{AKT}(1: 1,000)$, P-Akt $(1: 1,000)$, and $\beta$-actin $(1: 1,000)$ as a loading control. The membranes were subsequently incubated with HRP-conjugated anti-mouse IgG (Santa Cruz Biotechnology, Santa Cruz, CA, USA) or anti-eabbit IgG (Santa Cruz Biotechnology) for $2 \mathrm{hrs}$ at room temperature. Finally, immunoreactivity signal was visualized by Tanon Gel Imaging Systems (Shanghai, China).

\section{Statistical analysis}

SPSS (SPSS Inc., Chicago, IL, USA) for Windows (version 19.0) was used for data management and statistical analysis. All data were expressed as mean \pm SEM. Statistical analyses were performed using an independent-sample $t$-test or a one-way ANOVA followed by post hoc multiple comparisons. A value of $P<0.05$ was deemed to indicate statistical significance.

\section{Results}

TMP improved multiterritory perforator flap survival area and reduced tissue edema

After flap surgery, all rats survived without any postoperative infection. The boundary between survival and necrosis areas in the flap was clearly demarcated on postoperative day 7. The percentages of surviving flap areas in the control and TMP groups are shown in Figure 1. The mean surviving areas were $88.17 \pm 2.25 \%$ and 64.37 


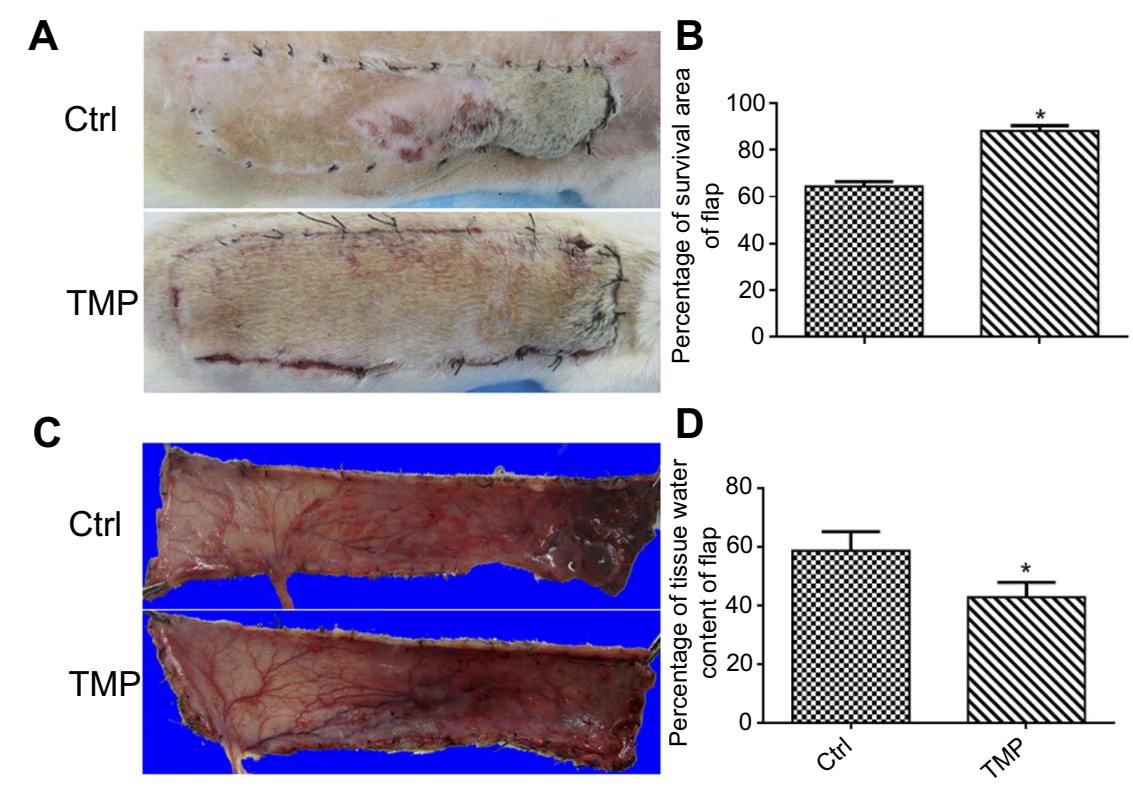

Figure I TMP improved multiterritory perforator flap survival and reduced tissue edema. (A) Image of the skin flaps taken on postoperative day 7. (B) Image of the inner sides of the skin flaps taken on day 7 to measure edema. (C) Survival percentages. (D) Tissue water content. Values are mean \pm SEM, $n=6$ per group. Significant differences between the groups are indicated by $* P<0.001$.

Abbreviations: Ctrl, control; TMP, tetramethylpyrazine.

$\pm 2.09 \%$ in the TMP treatment and control groups, respectively. Flap survival area was significantly higher in the TMP treatment group than in the control group (Figure 1A and $\mathrm{B} ; p<0.001)$. Percent tissue water content was significantly lower in the TMP treatment group (42.98 $\pm 2.06 \%)$ than in the control group $(58.87 \pm 2.65 \%$; Figure $1 \mathrm{C}$ and D; $P<0.001$ ), indicating that TMP treatment could reduce tissue edema.

\section{TMP-attenuated apoptosis in the ischemic area of flap}

To test whether TMP modulated cellular apoptosis in the ischemic area of the flap, the levels of Bax and Bcl-2 were analyzed by Western blot, and the TUNEL test was performed. The results of TUNEL staining are shown in Figure $2 \mathrm{~A}$ and $\mathrm{B}$, and the data presented that the number of apoptotic cells was significantly higher in the control group. By contrast, tissues obtained from rats treated with TMP revealed lesser apoptotic cells. Moreover, Western blot analysis results showed that the level of proapoptotic protein Bax was significantly downregulated, while antiapoptotic protein $\mathrm{Bcl}-2$ was significantly upregulated. The results data also demonstrated that the ratio of Bax/Bcl-2 was decreased after treatment with TMP (Figure 2B and C). All of these results demonstrated that TMP administration was an effective therapeutic strategy on inhibition of apoptosis in the ischemic area of the flap.

\section{TMP promoted angiogenesis in the ischemic area of flap}

On histopathological examination, the skin flaps exhibited more neovascularization and less necrosis than control flaps in the TMP treatment group (Figure 3A). The microvessel densities (MVDs) in the two groups from the results of $\mathrm{H} \& \mathrm{E}$ staining were $25.17 \pm 2.3$ and $10 \pm 1.63$, respectively (Figure $3 \mathrm{~B} ; p<0.001)$. CD31 is commonly used to label endothelial cells. Thus, the MVDs in the two groups were also reflected directly by the number of CD31-positive vessels. As shown in Figure 3C, the results demonstrated that TMP treatment increased the number of CD31-positive vessels in the multiterrory skin flap model: there were $25.67 \pm 1.8$ in the TMP group and $9.16 \pm 1.13$ in the control group (Figure 3D; $p<0.001$ ). The results of a whole-body angiography demonstrated that the microvascular imaging range of Area II and Area III was significantly greater in the TMP treatment group than in the control group. The results showed that the choke vessel zone I had higher dilation and the iliolumbar artery had increased its territory to supply adjacent vascular territories in the TMP group compared with that in the control group. Arteriography also demonstrated that choke vessel zone II dilated extensively in the TMP treatment group, whereas that in the control group dilated only slightly or remained unopened (Figure 3E). We also further assessed neovascularizationassociated factor (VEGF) by Western blotting analysis and 
A
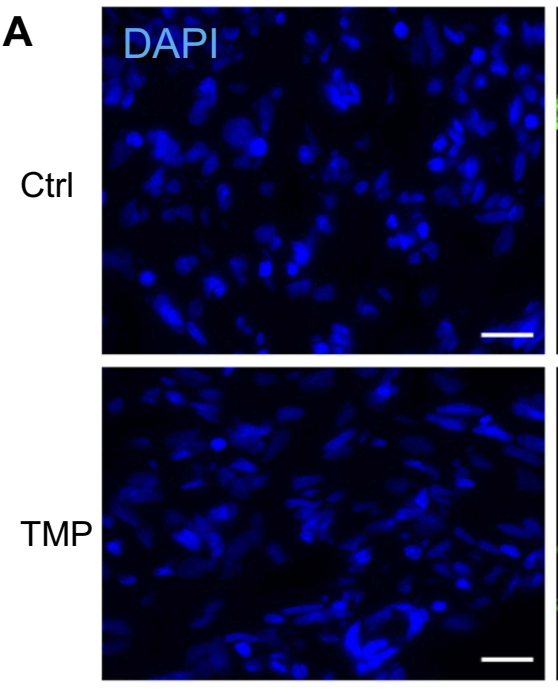

B

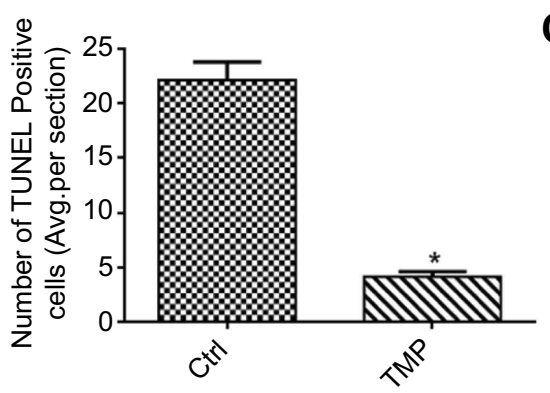

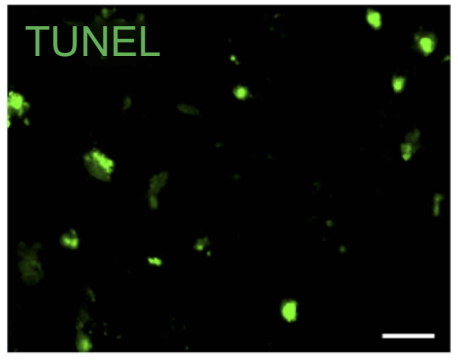

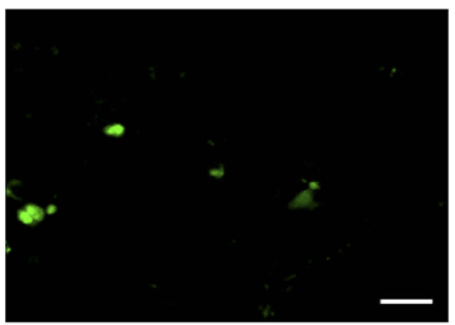

C

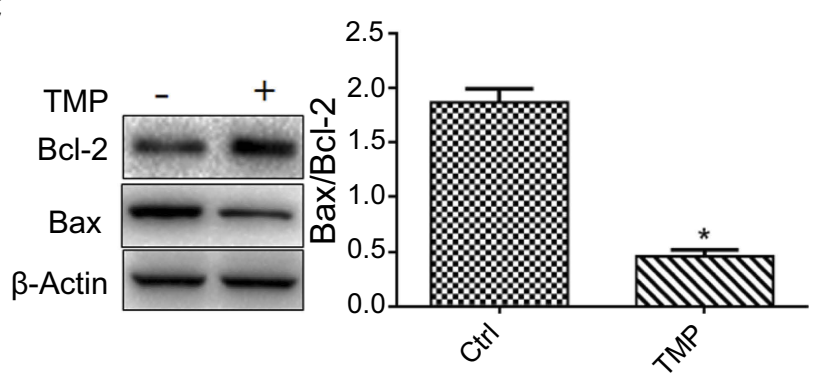

Figure 2 TMP attenuated apoptosis in the ischemic area of the flap. (A) TUNEL staining to assess the apoptosis. Scale bars are $10 \mu \mathrm{m}$. (B) Quantitative estimation of apoptotic and TUNEL cells. (C) Representative Western blots and quantification data of Bax, Bcl-2, and $\beta$-actin in each group; the data was represented with mean \pm SEM, $* P<0.05$ versus the control group, $\mathrm{n}=6$.

Abbreviations: Ctrl, control; TMP, tetramethylpyrazine.

found that the level of VEGF protein was significantly higher in the both TMP groups than in the control group (Figure 3F; $P<0.001$ ).

\section{TMP improved the survival of multiterritory perforator flap involving in the Akt/Nrf2 signaling pathway}

To investigate the role of the Akt/Nrf2 signaling pathway in TMP-associated flap vitality, the levels of p-Akt, Akt, and Nrf2 were analyzed by Western blot. The results demonstrated that the level of phosphorylation of Akt protein was significantly higher in the TMP groups than in the control group and TMP + LY294002 group (Figure $4 \mathrm{~A}$ and $\mathrm{B} ; P<0.05)$. The levels of Nrf2 protein also were significantly upregulated in the TMP group compared with that in the control group. Moreover, inhibition the Akt protein activities could downregulate the expression level of Nrf2 protein (Figure 4C; $P<0.05$ ).

To further confirm that TMP promoted angiogenesis and depressed apoptosis by activating the Akt/Nrf2 signaling pathway, the levels of VEGF, Bax, and Bcl-2 were detected by Western blot analysis and the MVDs were measured by the IHC and the TUNEL test was performed. The results indicated that inhibition of Akt protein activities could reduce the angiogenesis (Figure 4D and $\mathrm{E}$ ) and downregulated the expression of VEGF protein (Figure 4H). Similarly, the level of proapoptotic protein Bax was upregulated, while antiapoptotic protein $\mathrm{Bcl}-2$ was downregulated, and the ratio of Bax/Bcl-2 was increased after treatment with the AKT inhibitor LY294002 (Figure 4G). The results of TUNELS staining show that the number of apoptotic cells was significantly higher in the TMP + LY294002 group (Figure 4F). These results suggested that TMP may activate Akt/Nrf2 signaling pathway to induce the angiogenesis and attenuate the apoptosis in the ischemic area of the flap. We also found that inhibiting Akt protein activities reversed the effects of TMP on flap vitality (Figure 5). Taken together, these data revealed extensive activation of the Akt/Nrf2 signaling pathway in response to TMP in the ischemic area of the flap. Administration of the AKT inhibitor LY294002 reversed TMP-associated flap vitality. 


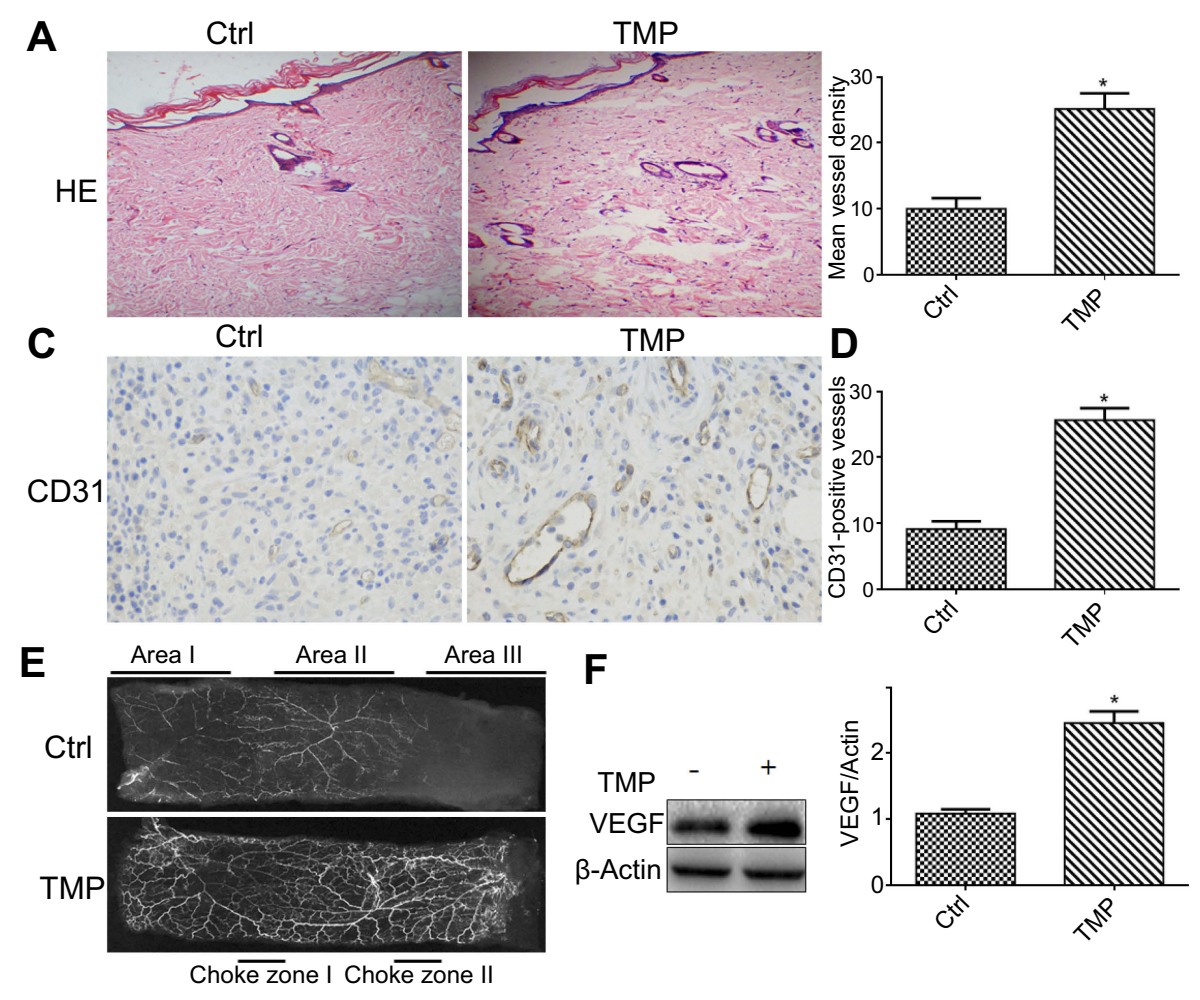

Figure 3 TMP promoted angiogenesis in the ischemic area of the flap. (A) On postoperative day 7, angiogenesis in the ischemic flap areas was evident by H\&E staining (original magnification $\times 100$ ). (B) MVDs assessed by H\&E staining. (C) Immunochemical detection of CD3I, which is a marker of angiogenesis (original magnification $\times 400$ ). (D) The numbers of CD3I-positive vessels. Values are mean \pm SEM, $n=6$ per group. Significant differences between the groups are indicated by $* P<0.00 \mathrm{I}$. (E) Postoperative angiography showed vascular territory of area I-III. Significant enhancement in dilation of choke vessels was observed in the second choke zone II. The vasculature was invisible in the necrosis area. (F) Representative Western blots and quantification data of VEGF and $\boldsymbol{\beta}$-actin in each group; Columns represent mean \pm SEM, $* P<0.05$ versus the control group.

Abbreviations: Ctrl, control; MVD, microvessel density; TMP, tetramethylpyrazine.

\section{Discussion}

TMP, the principal bioactive components of Ligusticum wallichii Franchat, exerts beneficial effects in patients with ischemic diseases such as neurovascular and cerebral ischemia/reperfusion injuries. ${ }^{22,23}$ Those previous studies have well-demonstrated that TMP had various biological activities such as proangiogenic, antioxidative and antiapoptotic activities. ${ }^{17,24,25}$ However, whether TMP treatment could address the effects in the skin flap necrosis by promoting angiogenesis and the apoptotic regulation remain to be elucidated. In the present study, we found that TMP significantly improved the multiterritory perforator flap survival, which was associated with enhancing angiogenesis and attenuating apoptosis.

Previous studies showed that choke vessels played a very important role in the distal partial survival of mutitlterritory perforator flaps. ${ }^{6,21}$ Unfortunately, the choke vessels of choke zone II often could not dilate or remodel timely which could not deliver oxygen and nutrients to the distal part of the skin flaps, and then result in the necrosis. In this study, one interesting phenomenon we observed was that the choke vessels in the choke zone II were dilated extensively in the TMP group, whereas those in the control group dilated only slightly or remained unopened. The results illustrated that TMP improves the survival area of the skin flap through accelerating the choke vessel remodeling.

Several reports indicated that TMP was proangiogenic both in vitro and in vivo. ${ }^{16,19,26}$ However, some authors also have claimed that TMP exerted antiangiogenic effects in models of liver fibers ${ }^{27}$ and several kinds of cancers.${ }^{28,29}$ Therefore, the effects of TMP treatment on angiogenesis remain controversial and may depend on the disease microenvironment. The effects of TMP treatment on multiterritory perforator flap angiogenesis remain unknown. In our present study, the MVD data which derived from H\&E staining and CD31 measurements demonstrated that TMP promoted neovascularization of skin flaps. We also further assessed neovascularization-associated factor (VEGF) by Western blotting analysis and found that the expression levels of VEGF upregulated in the TMP treatment group. Therefore, we inferred that TMP could promote the 

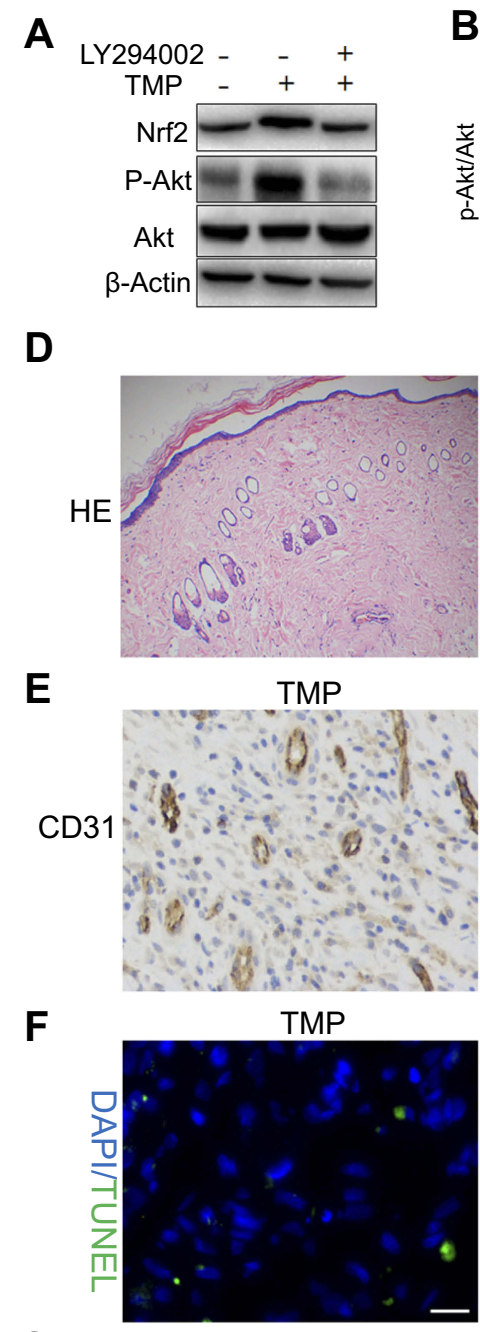

G

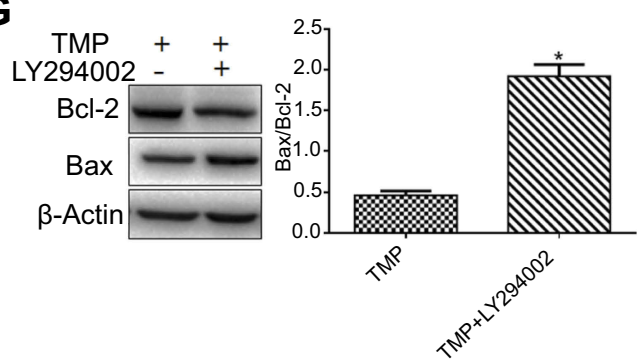

B
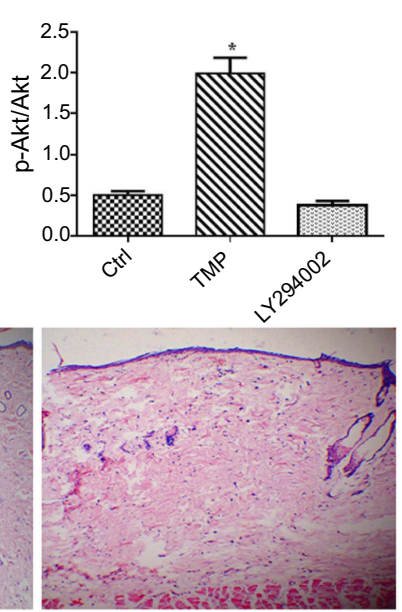

TMP+LY294002

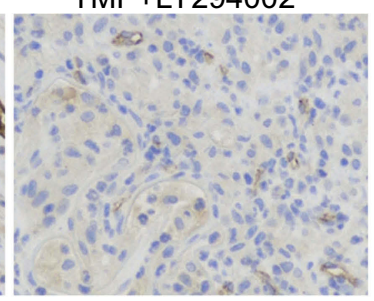

TMP+LY294002

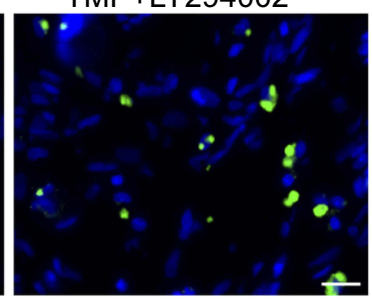

H
C
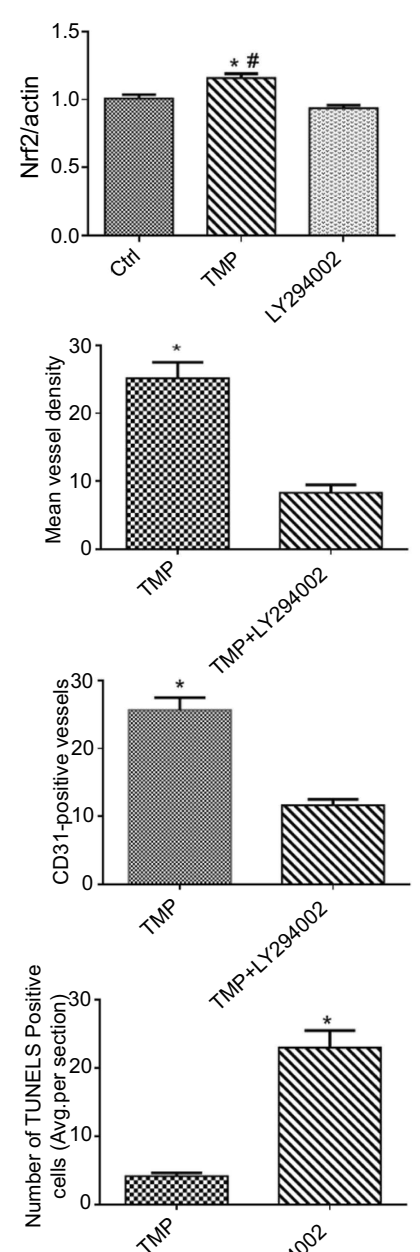
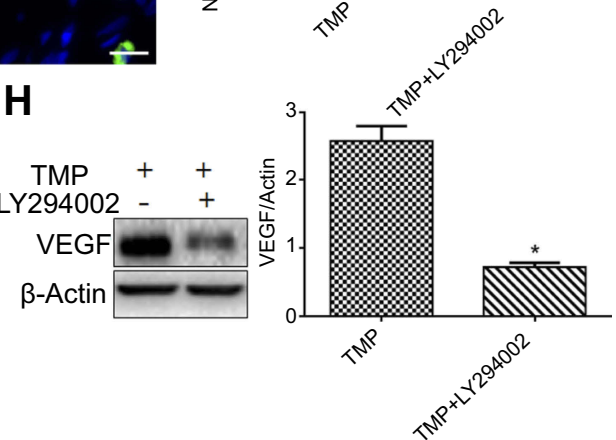

Figure 4 TMP improved the survival of skin flap via the Akt/Nrf2 signaling pathway. (A) The expressions of Akt, p-Akt, and Nrf2 were assessed by Western blot analysis. (B and C) Quantification data of Akt, P-Akt, Nrf2, and $\beta$-actin in each group; columns represent mean \pm SEM, *P<0.05 versus the control group or TMP + LY294002 group; $\# P<0.01$ versus the control group. (D) Neovascularization in the TMP and TMP + LY294002 groups by H\&E staining (magnifcation $\times 100)$. Columns represent mean \pm SEM, $* P<0.05$ versus the TMP + LY294002 group. (E) CD3I-positive vessels in the TMP and TMP + LY294002 groups as assessed by immunohistochemistry (magnifcation $\times 400$ ). Columns represent mean \pm SEM, $* P<0.05$ versus the TMP + LY294002 group. (F) TUNEL staining to assess the apoptosis at 7 days. Columns represent mean \pm SEM, $* P<0.05$ versus the TMP group. Scale bars are $10 \mu \mathrm{m}$. (G and $\mathbf{H}$ ) The expressions of angiogenesis-related protein VEGF and apoptosis-related protein Bax, Bcl-2 in each group by Western blot analysis. Columns represent mean \pm SEM, $* P<0.05$ versus the TMP group.

Abbreviation: TMP, tetramethylpyrazine.

viability of skin flap by upregulating VEGF-induced angiogenesis.

Ischemia/reperfusion injury and microcirculatory dysfunction were the principle reasons to impair the viability of the skin flap. ${ }^{30}$ The process of injury involved the formation of oxygen free radicals, oxidative stress and apoptosis. Apoptosis and oxidative stress induced by free radicals are the principal contributors to necrosis via the intrinsic mitochondrial pathway. Apoptosis is a process of programmed cell death that is regulated by signal transduction pathway. Bcl-2 and Bax are key proteins involved in this pathway. Among them, Bcl-2 is the protein with an 
A

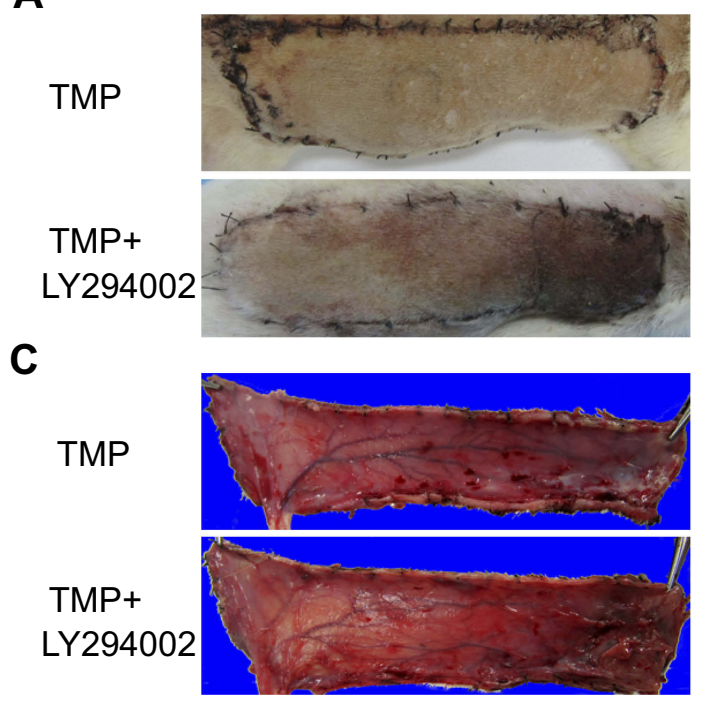

B

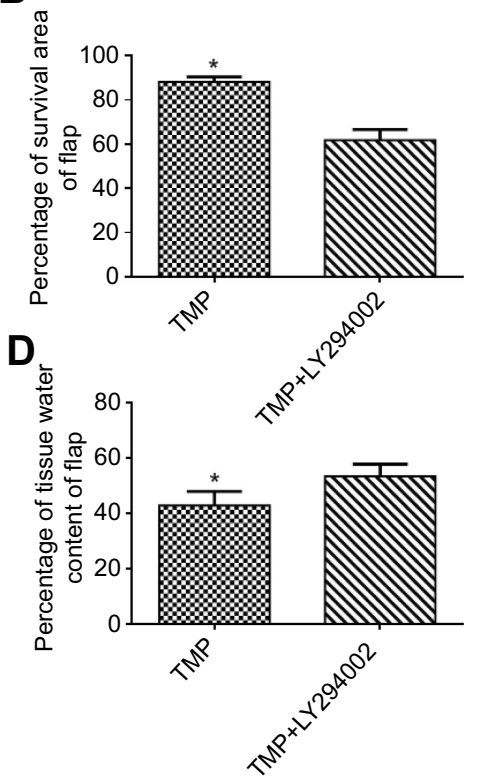

Figure 5 Inhibition of Akt protein activities reverse the effects of TMP on flap vitality. (A) Image showed the postoperative flaps of the TMP and TMP + LY294002 group on day 7. (B) The percentages of survival area in the TMP group (88.17 $\pm 2.25 \%$ ) and TMP + LY294002 group $(61.87 \pm 4.76 \%)$. Columns represent mean \pm SEM, $* P<0.001$ versus the TMP + LY294002 group. (C) Digital photographs presented the tissue edema of postoperative flaps of each group on day 7 . (D) The percentages of tissue water content. Columns represent mean \pm SEM, $* P<0.001$ versus the TMP + LY294002 group.

Abbreviation: TMP, tetramethylpyrazine.

antiapoptotic effect, and Bax participates in the induction of apoptosis. We assessed the effects of TMP on apoptosis during ischemia of mutitlterritory perforator flaps. Our results showed that the ratio of $\mathrm{Bax} / \mathrm{Bcl}-2$ was dramatically reduced in the TMP treatment group, which implied that the signal transduction pathway of apoptosis was suppressed by TMP. Further, DNA fragmentation was detected by TUNEL staining, and the results indicated that the numbers of TUNEL-positive cells were decreased in TMP-treated group compared with the control group, so we inferred that TMP could attenuate apoptosis in ischemia necrosis area of the multiterritory perforator flap.

Emerging studies reported that the pleiotropic effects of TMP were mediated by the activation of Nrf2 protein, which had been demonstrated to play an important role in antioxidative stress, antiapoptosis and proangiogenesis. ${ }^{31-33}$ Some literatures also described that Nrf2 protein could be activated by the phosphorylating Akt. ${ }^{34}$ In focal cerebral ischemia, it was proved that the stimulation of the PI3K/ Akt pathway was neuroprotective against hypoxic neuronal death in vitro and ischemic neuronal death in vivo, and there was increasing evidence to indicate cross-talk between the $\mathrm{Nrf2}$ and PI3K/Akt pathways in response to ischemia caused cell injury. ${ }^{16,35}$ Chen et $\mathrm{al}^{17}$ reported that TMP protected against ischemic stroke injury possibly in part through regulation of PGC1 $\alpha / \mathrm{Nrf} 2$ and PI3K/Akt pathways. Hence, we hypothesized that the effect of TMP treatment on improving the angiogenic and antiapoptosis might be involved in the Akt/Nrf2 pathway. Our data demonstrated that the levels of phosphorylation of Akt and Nrf2 protein were significantly higher in the TMP group than in the control group. Moreover, inhibiting Akt protein activities by the AKT inhibitor could reverse the effects of TMP on flap vitality. In addition, we also found that the use of the AKT inhibitor could disturb the effects of TMP on pro-angiogenesis and antiapoptosis. Thus, TMP could improve the viability of the skin flap through activation of the Akt/Nrf2 signaling pathway.

Of note, the limitations of TMP in multiterritory perforator flap therapy still need to be further investigated in the future study. For example, the optimal concentration postoperation administration should be demonstrated. In addition, the eventual side effects of the TMP treatment should also be identified in further study. Moreover, for further translational research, application in large animal model and humans will be beneficial; therefore, the study which is performed in large animal's model and human would need to further verify the beneficial effect of TMP on the flaps survival in the future.

In conclusion, this study detected the effects of TMP treatment on the viability of skin flap, and the results demonstrated that TMP treatment could attenuate the cell apoptosis and promote neovascularization in the 
multiterritory perforator flap. This process might be involved in the Akt/Nrf2 signaling pathway. Our results indicated that TMP might provide potential therapeutic intervention for improving the multiterritory perforator flap survival.

\section{Acknowledgments}

This research was supported in part by National Natural Science Foundation of China (No. 81472104). The research also was funded by Hunan Provincial Natural Science Foundation of China (No. 2018JJ2640).

\section{Disclosure}

The authors report no conflicts of interest in this work.

\section{References}

1. Tang J, Fang T, Song D, Liang J, Yu F, Wang C. Free deep inferior epigastric artery perforator flap for reconstruction of soft-tissue defects in extremities of children. Microsurgery. 2013;33(8):612619. doi: $10.1002 /$ micr.22127.

2. Zeltzer AA, Van Landuyt K. Reconstruction of a massive lower limb soft-tissue defect by giant free DIEAP flap. J Plast Reconstr Aesthet Surg. 2012;65(2):e42-45. doi:10.1016/j.bjps.2011.09.046

3. Qing L, Wu P, Yu F, Zhou Z, Tang J. Use of dual-skin paddle anterolateral thigh perforator flaps in the reconstruction of complex defect of the foot and ankle. J Plast Reconstr Aesthet Surg. 2018 doi:10.1016/j.bjps.2018.05.029

4. Qing L, Wu P, Liang J, Yu F, Wang C, Tang J. Use of flow-through anterolateral thigh perforator flaps in reconstruction of complex extremity defects. $J$ Reconstr Microsurg. 2015;31(8):571-578. doi:10.1055/s-0035-1555138

5. Tang J, Fang T, Song D, Liang J, Yu F, Wang C. Free deep inferior epigastric artery perforator flap for reconstruction of soft-tissue defects in extremities of children. Microsurgery. 2013;33(8):612619. doi:10.1002/micr.22127

6. Hamilton K, Wolfswinkel EM, Weathers WM, et al. The delay phenomenon: a compilation of knowledge across specialties. Craniomaxillofac Trauma Reconstr. 2014;7(2):112-118. doi:10.1055/s-0034-1371355

7. Lin R, Lin J, Li S, et al. Effects of the traditional Chinese medicine baicalein on the viability of random pattern skin flaps in rats. Drug Des Devel Ther. 2018;12:2267-2276. doi:10.2147/DDDT.S173371

8. Lin R, Chen H, Callow D, et al. Multifaceted effects of astragaloside IV on promotion of random pattern skin flap survival in rats. $A m J$ Transl Res. 2017;9(9):4161-4172.

9. Zhou KL, Zhang YH, Lin DS, Tao XY, Xu HZ. Effects of calcitriol on random skin flap survival in rats. Sci Rep. 2016;6:18945. doi: $10.1038 /$ srep 18945

10. Gill PS, Hunt JP, Guerra AB, et al. A 10-year retrospective review of 758 DIEP flaps for breast reconstruction. Plast Reconstr Surg. 2004;113(4):1153-1160.

11. Wang L, Zhou ZW, Yang LH, et al. Vasculature characterization of a multiterritory perforator flap: an experimental study. $J$ Reconstr Microsurg. 2017;33(4):292-297. doi:10.1055/s-0036-1598011

12. Wang L, Jin Z, Wang J, et al. Detrimental effect of Hypoxia-inducible factor-1alpha-induced autophagy on multiterritory perforator flap survival in rats. Sci Rep. 2017;7(1):11791. doi:10.1038/s41598-017-12034-x
13. Yun I, Lew DH, Kim YS, et al. The effect of omeprazole usage on the viability of random pattern skin flaps in rats. Wound Repair Regener. 2017;78(6):e5-e9.

14. Wang LR, Cai LY, Lin DS, Cao B, Li ZJ. Effect of electroacupuncture at the zusanli point (stomach-36) on dorsal random pattern skin flap survival in a rat model. Dermatologic Surg. 2017;43(10):12131220. doi:10.1097/DSS.0000000000001178

15. Li K, Wang L, Rehman MU, et al. Preconditioning of bone marrowderived mesenchymal stromal cells by tetramethylpyrazine enhances cell migration and improves functional recovery after focal cerebral ischemia in rats. Sci Rep. 2017;8(1):112.

16. Hu JZ, Wang XK, Cao Y, et al. Tetramethylpyrazine facilitates functional recovery after spinal cord injury by inhibiting MMP2, MMP9, and vascular endothelial cell apoptosis. Curr Neurovasc Res. 2017;14(2):110-116. doi:10.2174/1567202614666170313 114115

17. Chen H, Cao J, Zhu Z, et al. A novel tetramethylpyrazine derivative protects against glutamate-induced cytotoxicity through PGC1alpha/ Nrf2 and PI3K/Akt signaling pathways. Front Neurosci. 2018;12:567. doi:10.3389/fnins.2018.00044

18. Lu C, Zhang J, Shi X, et al. Neuroprotective effects of tetramethylpyrazine against dopaminergic neuron injury in a rat model of Parkinson's disease induced by MPTP. Int J Biol Sci. 2014;10 (4):350-357. doi:10.7150/ijbs.8366

19. Wang C, Li Y, Yang X, et al. Tetramethylpyrazine and astragaloside IV synergistically ameliorate left ventricular remodeling and preserve cardiac function in a rat myocardial infarction model. $J$ Cardiovasc Pharmacol. 2017;69(1):34-40. doi:10.1097/FJC.000 0000000000437

20. Du W, Wu PF, Qing LM, et al. Systemic and flap inflammatory response associates with thrombosis in flap venous crisis. Inflammation . 2015;38(1):298-304. doi:10.1007/s10753-0140033-9

21. Qing L, Lei P, Tang J, et al. Inflammatory response associated with choke vessel remodeling in the extended perforator flap model. Exp Ther Med. 2017;13(5):2012-2018. doi:10.3892/ etm.2017.4205

22. Ho WK, Wen HL, Lee CM. Tetramethylpyrazine for treatment of experimentally induced stroke in Mongolian gerbils. Stroke. 1989;20 (1):96-99.

23. Xiao X, Liu Y, Qi C, et al. Neuroprotection and enhanced neurogenesis by tetramethylpyrazine in adult rat brain after focal ischemia. Neurol Res. 2010;32(5):547-555. doi:10.1179/17431320 9X414533

24. Wang C, Wang P, Zeng W, Li W. Tetramethylpyrazine improves the recovery of spinal cord injury via Akt/Nrf2/HO-1 pathway. Bioorg Med Chem Lett. 2016;26(4):1287-1291. doi:10.1016/j. bmcl.2016.01.015

25. Gong X, Ivanov VN, Hei TK. 2,3,5,6-Tetramethylpyrazine (TMP) down-regulated arsenic-induced heme oxygenase-1 and ARS2 expression by inhibiting Nrf2, NF-kappaB, AP-1 and MAPK pathways in human proximal tubular cells. Arch Toxicol. 2016;90 (9):2187-2200. doi:10.1007/s00204-015-1600-z

26. Wang Y, Guo G, Yang BR, et al. Synergistic effects of ChuanxiongChishao herb-pair on promoting angiogenesis at network pharmacological and pharmacodynamic levels. Chin J Integr Med. 2017;23 (9):654-662. doi:10.1007/s11655-017-2408-x

27. Cai X, Chen Z, Pan X, et al. Inhibition of angiogenesis, fibrosis and thrombosis by tetramethylpyrazine: mechanisms contributing to the SDF-1/CXCR4 axis. PLoS One. 2014;9(2):e88176. doi:10.1371/journal.pone. 0088176

28. Jia Y, Wang Z, Zang A, Jiao S, Chen S, Fu Y. Tetramethylpyrazine inhibits tumor growth of lung cancer through disrupting angiogenesis via BMP/Smad/Id-1 signaling. Int J Oncol. 2016;48(5):2079-2086. doi:10.3892/ijo.2016.3443 
29. Tang JH, Zhang HM, Zhang ZH, Zhang XL. Effect of tetramethylpyrazine combined with cisplatin on VEGF, KLF4 and ADAMTS1 in Lewis lung cancer mice. Asian Pac J Trop Med. 2017;10(8):813-818. doi:10.1016/j.apjtm.2017.08.001

30. Harder Y, Amon M, Erni D, Menger MD. Evolution of ischemic tissue injury in a random pattern flap: a new mouse model using intravital microscopy. J Surg Res. 2004;121(2):197-205. doi:10.1016/j. jss.2004.03.026

31. Bai Q, Lyu Z, Yang X, Pan Z, Lou J, Dong T. Epigallocatechin-3gallate promotes angiogenesis via up-regulation of $\mathrm{Nfr} 2$ signaling pathway in a mouse model of ischemic stroke. Behav Brain Res. 2017;321:79-86. doi:10.1016/j.bbr.2016.12.037

32. Abdelsalam RM, Safar MM. Neuroprotective effects of vildagliptin in rat rotenone Parkinson's disease model: role of RAGE-NFkappaB and Nrf2-antioxidant signaling pathways. J Neurochem. 2015;133 (5):700-707. doi:10.1111/jnc.13087
33. Aliaghaei A, Khodagholi F, Ahmadiani A. Conditioned media of choroid plexus epithelial cells induces Nrf2-activated phase II antioxidant response proteins and suppresses oxidative stress-induced apoptosis in PC12 cells. J Mol Neurosci. 2014;53(4):617-625. doi:10.1007/s12031-014-0228-4

34. Chen NN, Wang JP, Liu HF, et al. The bone marrow mononuclear cells reduce the oxidative stress of cerebral infarction through PI3K/AKT/NRF2 signaling pathway. Eur Rev Med Pharmacol Sci. 2017;21(24):5729-5735. doi:10.26355/ eurrev 20171214019

35. Hu J, Cao Y, Wu T, Li D, Lu H. Micro-CT as a tool to investigate the efficacy of tetramethylpyrazine in a rat spinal cord injury model. Spine. 2016;41(16):1272-1278. doi:10.1097/BRS.0000000000 


\section{Supplementary material}
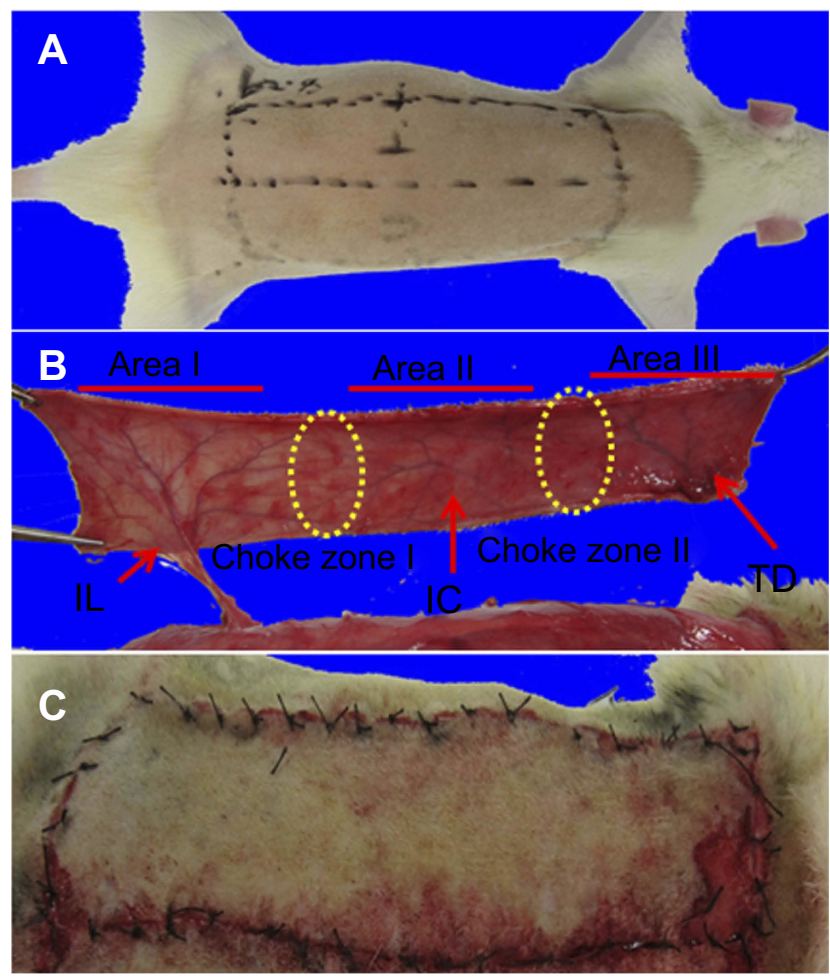

Figure SI (A) The dorsal multiterritory skin flap measuring $4 \times 10 \mathrm{~cm}$ was designed. (B and C) The flap was completely detached from the surrounding skin, depending only on the iliolumbar vessels for perfusion and drainage.

Abbreviations: TD, thoracodorsal vessels; IC, posterior intercostal vessels; IL, iliolumbar perforator.

\section{Publish your work in this journal}

Drug Design, Development and Therapy is an international, peerreviewed open-access journal that spans the spectrum of drug design and development through to clinical applications. Clinical outcomes, patient safety, and programs for the development and effective, safe, and sustained use of medicines are a feature of the journal, which has also been accepted for indexing on PubMed Central. The manuscript management system is completely online and includes a very quick and fair peer-review system, which is all easy to use. Visit http://www. dovepress.com/testimonials.php to read real quotes from published authors. 